\title{
The mazEF toxin-antitoxin system as an attractive target in clinical isolates of Enterococcus faecium and Enterococcus faecalis
}

This article was published in the following Dove Press journal:

Drug Design, Development and Therapy

8 May 2015

Number of times this article has been viewed

\author{
Sara Soheili' \\ Sobhan Ghafourian² \\ Zamberi Sekawi' \\ Vasantha Kumari Neela' \\ Nourkhoda Sadeghifard ${ }^{2}$ \\ Morovat Taherikalani ${ }^{2}$ \\ Afra Khosravi ${ }^{2}$ \\ Ramliza Ramli ${ }^{3}$ \\ Rukman Awang Hamat \\ 'Department of Medical Microbiology \\ and Parasitology, Faculty of Medicine \\ and Health sciences, Universiti Putra \\ Malaysia, Serdang, Malaysia; ${ }^{2}$ Clinical \\ Microbiology Research Center, \\ Ilam University of Medical Sciences, \\ Ilam, Iran; ${ }^{3}$ Department of Medical \\ Microbiology and Immunology, Faculty \\ of Medicine, Universiti Kebangsaan \\ Malaysia Medical Centre, Jalan Yaakob \\ Latif, Bandar Tun Razak, Kuala Lumpur, \\ Malaysia
}

\begin{abstract}
The toxin-antitoxin (TA) system is a regulatory system where two sets of genes encode the toxin and its corresponding antitoxin. In this study, the prevalence of TA systems in independently isolated clinical isolates of Enterococcus faecium and Enterococcus faecalis was determined, the dominant TA system was identified, different virulence genes in E. faecium and $E$. faecalis were surveyed, the level of expression of the virulence and TA genes in normal and stress conditions was determined, and finally their associations with the TA genes were defined. Remarkably, the analysis demonstrated higBA and $m a z E F$ in all clinical isolates, and their locations were on chromosomes and plasmids, respectively. On the other hand, a quantitative analysis of TA and virulence genes revealed that the expression level in both genes is different under normal and stress conditions. The results obtained by anti-mazF peptide nucleic acids demonstrated that the expression level of virulence genes had decreased. These findings demonstrate an association between TA systems and virulence factors. The mazEF on the plasmids and the higBA TA genes on the chromosomes of all E. faecium and E. faecalis strains were dominant. Additionally, there was a decrease in the expression of virulence genes in the presence of anti-maz $F$ peptide nucleic acids. Therefore, it is suggested that mazEF TA systems are potent and sensitive targets in all E. faecium and E. faecalis strains.
\end{abstract}

Keywords: Enterococcus faecium, Enterococcus faecalis, toxin-antitoxin system, virulence genes, peptide nucleic acids

\section{Introduction}

Toxin-antitoxin (TA) systems, first described in the mid-1980s, are regulatory loci that encode a toxin and its corresponding antitoxin. The TA system could be RNA or a protein, but in all TA systems reported to date, the antitoxin has been found to be unstable while the toxin is stable. ${ }^{1}$ TA loci are often transferred by horizontal transformation and are more often associated with pathogenic bacteria; most of them have been found on plasmids containing antibiotic resistance, ${ }^{2}$ and virulence genes may also be harbored on TA plasmids. The use of toxins has been proposed as a new approach for antimicrobial therapy in pathogenic bacteria. ${ }^{3}$

The majority of clinical enterococcal infections are caused by Enterococcus faecalis and Enterococcus faecium. E. faecalis is considered to be more virulent; however, E. faecium is more likely to be antibiotic resistant. ${ }^{4}$ Twenty years ago, only $10 \%$ of the nosocomial enterococcal infections were caused by E. faecium. ${ }^{4}$ Now, approximately $40 \%$ of the enterococcal nosocomial infections worldwide are caused by E. faecium. ${ }^{5}$ This ratio changed in favor of E. faecium in the United States during the late 1990s and in Europe around the year 2000. ${ }^{6}$ In the last two decades, the emergence of enterococci as an important nosocomial pathogen has been
Correspondence: Rukman Awang Hamat Department of Medical Microbiology and Parasitology, Faculty of Medicine and Health Sciences, Universiti Putra Malaysia, 43400 Serdang, Selangor, Malaysia

Tel +60 389472369

Fax +60389413802

Email rukman@upm.edu.my 
increasingly documented. Unfortunately, the pathogenesis of enterococcal infections is only partly understood. However, several adhesins, hemolysin, hyaluronidase, aggregation substances, gelatinase, and genes encoding pili are now considered possible virulence factors. ${ }^{7}$ So far, at least 22 different genes, collectively called fms (E. faecium surface protein-encoding genes) are considered putative virulence factors in Enterococcus spp. Virulence factors encoded by acmfm (fms 8 ), $h y l$, espfm, sgrA, and ecbA are most strongly associated with clinical lineages in E. faecium. ${ }^{8}$ Antisense therapies, which are sequence dependent, silence a specific gene. The antisense components are analogs of messenger (m)RNA; therefore, this technology is involved in the inhibition of gene expression. Many techniques are available for antisense therapy that use different RNA analogs, such as phosphorodiamidate morpholino oligomers, locked nucleic acids, and peptide nucleic acids (PNA). Among these, the properties of PNA make them particularly appropriate for antisense therapy in bacteria. This technique is applied for molecular bioengineering, therapeutic methods, and antibiotics. ${ }^{9-11}$ The structure of PNAs is similar to that of DNA or RNA, except that the nucleobases are changed to a pseudopeptide ${ }^{12}$ following the Watson and Crick basepairing rule; however, PNAs can bind DNA and RNA. ${ }^{13}$

The TA system could be a potent target for antibiotic therapy. In theory, the activation of a toxin or the inhibition of an antitoxin is an attractive strategy for antimicrobial therapy. ${ }^{14,15}$ Amitai et $\mathrm{ll}^{16}$ demonstrated that $5 \%$ of bacterial cells were viable and 95\% were killed after toxin activation because the increased toxin could not be neutralized by the antitoxin. However, when coexpressing $\operatorname{maz} E$ (antitoxin) and neutralizing $\operatorname{maz} F$ (toxin), $85 \%$ of the cells were viable because the toxin was neutralized and inhibited by the antitoxin. ${ }^{16}$ Nonetheless, the most important step for potency of the TA system, as a target, is to identify a TA system that is prevalent in all pathogenic clinical strains and to determine its functionality. While the analysis of a TA system can be instructive, until now, there has been no information available on the prevalence and identity of TA systems in pathogenic E. faecium and E. faecalis. Therefore, it is necessary to study a TA system that is prevalent and transcribed in all clinical pathogenic E. faecium and E. faecalis strains and to evaluate the TA system as a potent target in E. faecium and E. faecalis.

\section{Materials and methods \\ Ethics statement}

The ethical committee members of the Universiti Putra Malaysia (Selangor, Serdang, Malaysia) approved (JMMPJuly [10] 02) the collection of wound samples for the identification of Enterococcus spp. from patients; all patients and the parents or guardians of children in this study provided their written consent. The ethical committee of the Universiti Putra Malaysia specifically approved this study.

\section{Bacterial isolates}

A total of 79 clinical isolates of E. faecalis and E. faecium were identified during the period of May 2009-March 2010 from a tertiary teaching hospital. Of these Enterococcus isolates, 29 were $E$. faecium and 50 isolates were $E$. faecalis. The isolates were collected from urine, blood, pus, vaginal, and sterile body fluids.

\section{Evaluation of different virulence genes}

All of the isolates were subjected to the amplified pilA, pilB, $h y l A, e c b A, s c m, f m s 8, e f a A f m$, and $s g r A$ genes using the specific primers that were designed.

\section{Evaluation of the TA systems}

All the clinical isolates of E. faecium and E. faecalis were subjected to polymerase chain reaction (PCR) using total chromosomal or plasmid DNA. Oligodeoxy nucleotide primer pairs were designed for specific genes using sequences obtained from GenBank (European Nucleotide Archive: http://www.ebi.ac.uk/ena/). Primers were synthesized to amplify the mazEF, relBE, and higBA TA genes.

\section{Plasmid transformation}

For confirmation that the TA genes were harbored by plasmids, a transformation was performed. The plasmid-free strain Staphylococcus aureus RN4220 was used as a transforming host. Plasmid DNA was extracted from the clinical isolates using a plasmid extraction kit (Thermo Fisher Scientific, Waltham, MA, USA) and was then fractionated into agarose gel by electrophoresis. Each plasmid-specific band was purified from the agarose gel using the QIAEX II ${ }^{\circledR}$ Gel Extraction Kit. The isolated plasmids were then transformed into the $S$. aureus RN4220 competent cells. For confirmation that the plasmids harbored the TA genes, different purified plasmids were subjected to PCR.

\section{Sequence analysis}

The PCR products of the virulence and TA genes were purified from gel agarose, and the purified products were sequenced by Sigma-Aldrich Co. (St Louis, MO, USA). The results of the DNA sequencing were run in the Chromas Lite program to analyze their similarity to the sequenced gene in the GenBank library. 


\section{Stress induction}

The stress on the E. faecium and E. faecalis strains was induced by heat stress aqua: E. faecium and E. faecalis were shifted from $37^{\circ} \mathrm{C}$ to $42.5^{\circ} \mathrm{C}$ for 30 minutes, as described by Anderson et al. ${ }^{17}$ RNA was extracted from the bacteria in normal and stress conditions, and the level of expression of the TA systems and virulence genes was evaluated by realtime quantitative (RT-q)PCR.

\section{RT-qPCR}

The specific primers and TaqMan probes for the TA genes, the virulence genes, and the $23 \mathrm{~S}$ ribosomal (r)RNA (a reference gene) were all designed using the GenScript software and were synthesized by Sigma-Aldrich Co. The ability of the primers for each specific gene to amplify the appropriate amplicon length was evaluated using $4 \mu \mathrm{L}$ of complementary (c)DNA in a total volume of $20 \mu \mathrm{L}$ per reaction in a Mastercycler ${ }^{\circledR}$ realplex 2 (Eppendorf, Hamburg, Germany) with the TaqMan ${ }^{\circledR}$ Fast Universal PCR Master Mix. The thermocycling conditions consisted of an initial denaturation for 15 minutes at $95^{\circ} \mathrm{C}$, followed by 40 cycles of $95^{\circ} \mathrm{C}$ for 15 seconds, $60^{\circ} \mathrm{C}$ for 40 seconds and $68^{\circ} \mathrm{C}$ for 20 seconds. The RT-qPCR results were then analyzed quantitatively to estimate the level of TA and the virulence transcription factors in E. faecium and E. faecalis by calculating the number of mRNAs that were produced.

\section{PNA design}

The $m a z F$ toxin was targeted for the antisense PNA therapy. Specific PNA was designed for the $m a z F$ toxin. The $\operatorname{mazF}$ (+10 start codon) PNA sequence was designed and synthesized by Promega Corporation (Fitchburg, WI, USA). The PNA structure was mazF:KFFKFFKFFK-egATGATTAGAC-CONH2.

\section{Antisense PNA therapy of E. faecium and $E$. faecalis containing the mazEFTA locus plasmid}

A $0.5 \mathrm{McF}$ arland standard of clinical isolates of E. faecalis (three isolates) and E. faecium (two isolates) containing the plasmid $m a z E F$ TA locus and all the virulence genes were prepared. PNA of different concentrations $(1-10 \mu \mathrm{mol})$ in each well of a Costar microplate, in combination with $0.5 \mathrm{McFarland}$ standards of $E$. faecalis and $E$. faecium, were prepared. The negative control was Mueller Hinton broth, and the positive control was E. faecium without PNA. The Costar microplate was incubated at $35^{\circ} \mathrm{C}$. The effects of PNA at different concentrations $(1-10 \mu \mathrm{mol})$ against $E$. faecalis and
E. faecium were examined. RT-qPCR was performed to check the expression level of $\operatorname{maz} F$ and the virulence genes.

\section{Results and discussion The mazEF and higBATA genes are prevalent in all clinical isolates of E. faecium and $E$. faecalis}

In the current study, both plasmid and chromosomal DNA were subjected to amplification by PCR to identify the prevalence of different TA genes and their location on the plasmid or chromosome. For this purpose, after extraction of the chromosomal and plasmid DNA from the E. faecalis and $E$. faecium strains, the specific primers were used to amplify the $m a z E F$, relBE, and higBA TA genes. All chosen TA systems belong to a type II TA system; thus, both the toxin and antitoxin are proteins. For accuracy of the PCR results, the toxin and antitoxin were separately subjected to amplification. The results were confirmed with the presence of both the toxin and antitoxin genes. The results of the PCR analysis demonstrated that the TA genes were more prevalent on the plasmid than on the chromosome; the exception was the higBA TA system. The prevalence of the TA systems is displayed in Figure 1.

The mazEF TA system was found on the plasmids of all E. faecium and E. faecalis strains, but it was present in only $59.4 \%$ (number $[n]=43 / 79$ ) of the chromosomal DNA samples. It could be concluded that mazEF was likely harbored by the plasmid; this can be confirmed by plasmid transformation. Evaluation of the relBE TA system showed that $59.5 \%(\mathrm{n}=47 / 79)$ of the E. faecium and E. faecalis strains were positive for the relBE TA system on the plasmid. However, lower prevalence rates $(19 \%[\mathrm{n}=15 / 79])$ were observed in the chromosomal DNA. When evaluating $E$. faecium strains for the presence of higBA TA loci, 39.2\% $(\mathrm{n}=31 / 79)$ of the plasmid DNA and $100 \%(\mathrm{n}=79 / 79)$ of the chromosomal DNA possessed the higBA TA system.

Therefore, the mazEF and higBA TA loci had a $100 \%$ prevalence among $E$. faecium and $E$. faecalis. Thus, the $m a z E F$ and $h i g B A$ TA systems are putative targets that are prevalent in all E. faecium and E. faecalis strains.

After using the plasmid extraction kit, the plasmid was subjected to adenosine triphosphate-dependent DNase, which digested the linear DNA, but was not effective against doublestrand super-coiled DNA. This confirmed that there was no contamination in the chromosomal DNA. The confirmation was made through plasmid transformation into the $S$. aureus RN4220 free plasmid as well. The next section shows that the $m a z E F$ TA locus is harbored by a $20 \mathrm{~kb}$ plasmid. 


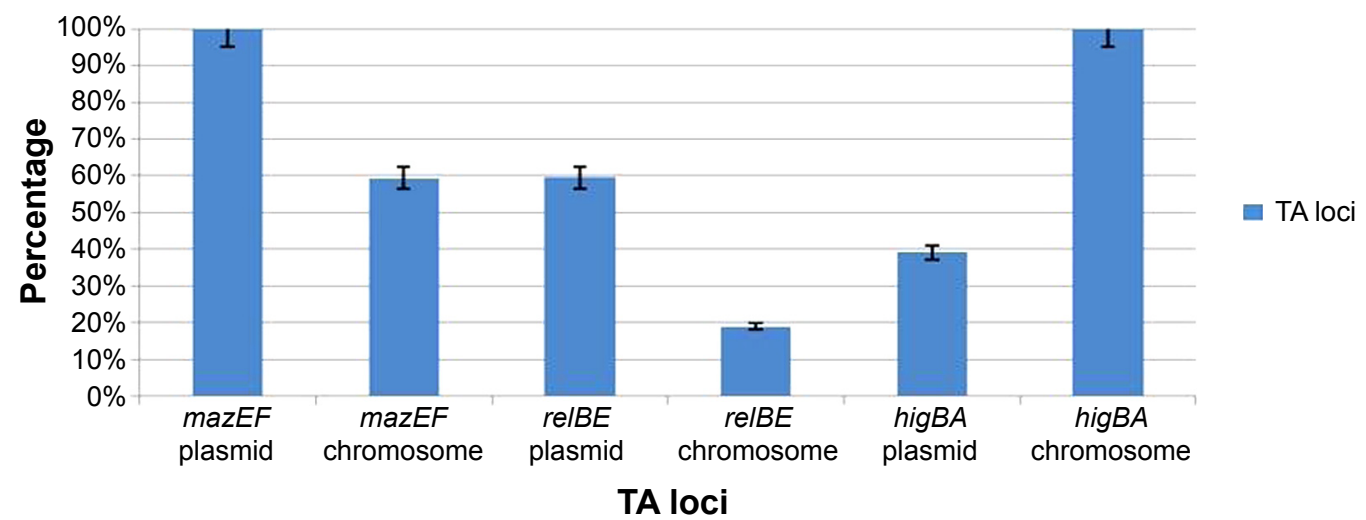

Figure I Prevalence of the different TA loci among Enterococcus faecium and Enterococcus faecalis clinical isolates. Abbreviation: TA, toxin-antitoxin.

\section{The mazEF TA system is harbored by a plasmid while the possible location of relBE is on the chromosome}

To confirm that the TA locus was harbored by a plasmid and not contaminated by the chromosomal DNA, a transformation was performed. After extraction of the plasmid from $E$. faecium using the plasmid extraction kit, the plasmids were fractionated on $1 \%$ agarose gel. Then, each plasmid was purified using the QIAEX II ${ }^{\circledR}$ Gel Extraction Kit, which is able to purify DNA fragments between $100 \mathrm{bp}$ and $50 \mathrm{~kb}$ in length. As shown in Figure 2, most E. faecium and E. faecalis strains contain two plasmids, while some of the strains contain three plasmids.

After purification of each plasmid, they were subjected to PCR to probe for the mazEF and higBA TA loci. The $20 \mathrm{~kb}$ plasmid had a positive result for $m a z E F$, while negative results were obtained for $h i g B A$ in all the purified plasmids. Thus, the $20 \mathrm{~kb}$ plasmid was transformed to the $S$. aureus RN4220 plasmid-free strain by electroporation. Plasmid extraction was performed on the $S$. aureus RN4220 containing the mazEF TA system plasmid (Figure 3). PCR was performed to confirm the presence of the mazEF TA locus on the $20 \mathrm{~kb}$ plasmid. These results confirmed that the mazEF TA system is harbored by the plasmid. The PCR results of the plasmid confirmed the presence of mazEF and the absence of higBA. Therefore, the mazEF TA locus is harbored by the plasmid, and the bacterial chromosome potentially possesses the higBA TA locus.

\section{pilB, fms8, efaAfm, and sgrA are the most prevalent virulence genes in $E$. faecium and $E$. faecalis}

A total of 79 E. faecalis (50 isolates) and E. faecium (29 isolates) isolates from clinical infections were analyzed for the presence of different virulence genes including pilA,
pilB, hylA, ecbA, scm, fms 8 , efaAfm, and sgrA. The analysis showed that the prevalence of the different virulence genes in Enterococcus spp. ranged from $35.4 \%$ to $100 \%$. pilB, fms 8 , efaAfm, and sgrA were the most prevalent virulence genes and were observed in all 79 isolates. The second most prevalent virulence gene, scm, was found in $92.4 \%$ of the isolates $(n=73 / 79)$. ecbA was determined to be the third most prevalent virulence gene with a frequency of $81 \%(\mathrm{n}=64 / 79)$. pilA had a prevalence of $73.4 \%(\mathrm{n}=58 / 79)$ in the clinical isolates of Enterococcus spp. The lowest prevalence was reported in $h y l$ with a frequency of $35.4 \%(n=28 / 79)$. The prevalence rates of the different virulence genes are shown in Figure 4. The analysis also showed that all the selected virulence genes were found in E. faecium and that hyl was predominant with a prevalence rate of $82.7 \%$. In E. faecalis, all the virulence genes with different prevalence rates were observed, as shown in Table 1. This is the first report of the prevalence of selected virulence genes in E. faecalis, which demonstrates the possibility of horizontal transformation between E. faecium and E. faecalis.

\section{Functionality of TA loci based on an evaluation of the quantity of toxin and antitoxin}

To assess the level of expression of $m a z E$ and $m a z F, ~ r e l B$ and relE, and $h i g B$ and $h i g A$, RT-qPCR was performed. The level of expression of each TA gene in stressed cultures was evaluated relative to the control cells. The experiment was compared with that under normal conditions. The results under normal conditions had almost the same quantity of toxin and antitoxin, as indicated in Figure 5. The results of the heat stress showed an increase in toxin levels and a decrease in antitoxin levels. The reason for this increase in toxin and decrease in antitoxin could be explained by the degradation of 


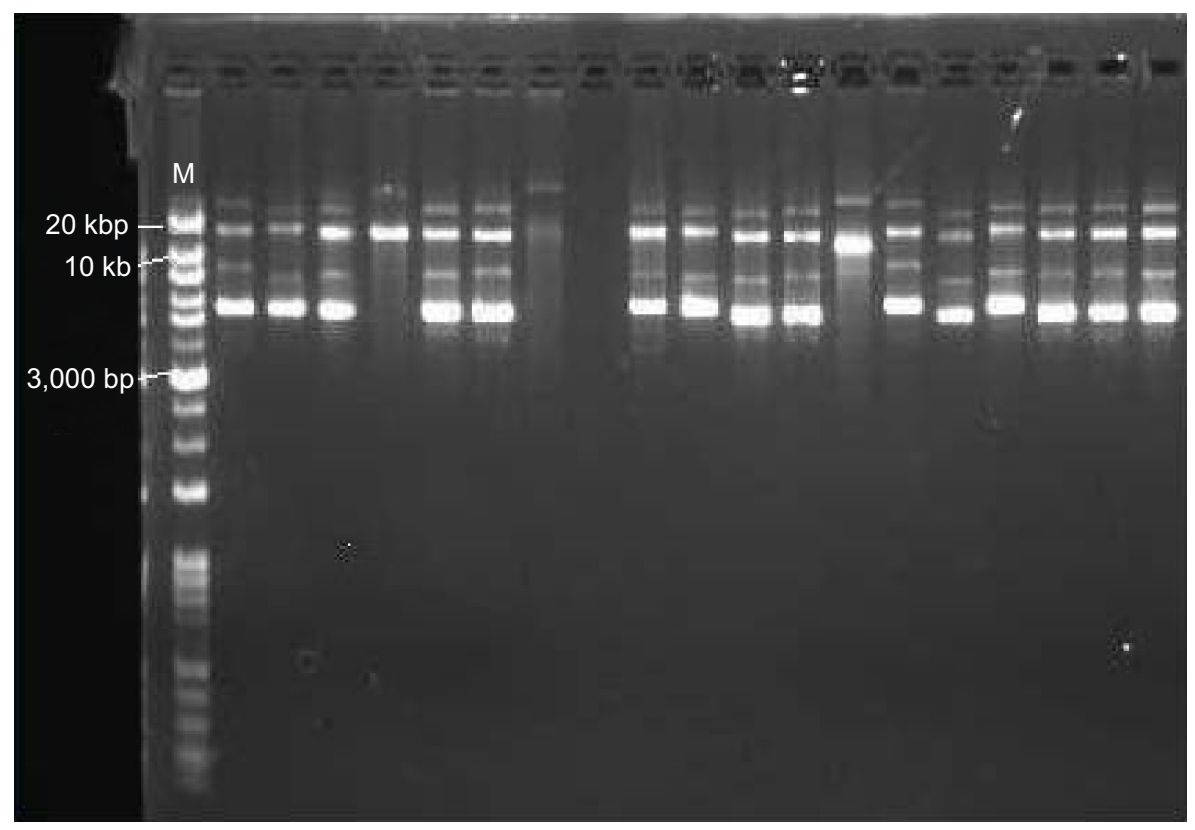

Figure 2 Plasmid profiles of different Enterococcus spp. strains.

Note: Lane M: marker.

the antitoxin. Greater transcription occurred because the TA system had the same promoter, and the antitoxin was unstable and was degraded by a protease. Therefore, the amount of toxin increased. However, the analysis by RT-qPCR showed that stress could increase the expression of toxin and decrease antitoxin expression.

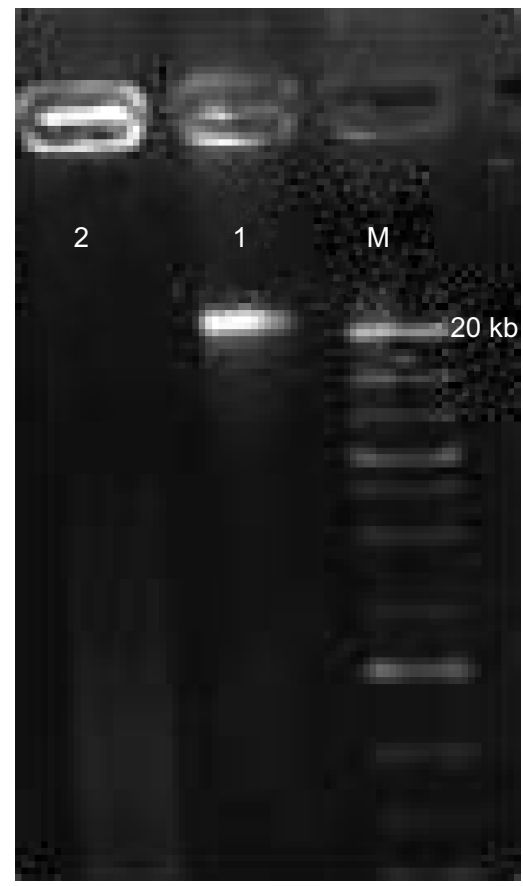

Figure 3 S. aureus RN4220 containing the mazEF TA locus on a plasmid after transformation.

Notes: Lane M: marker; lane I: 20 kb plasmid containing mazEF TA loci; lane 2: plasmid-free S. aureus RN4220 (negative control).

Abbreviations: S. aureus, Staphylococcus aureus; TA, toxin-antitoxin.
In 2009, Ramage et a $1^{18}$ demonstrated the functionality of the TA system under stress using RT-qPCR. They identified 88 different TA systems in Mycobacterium tuberculosis. ${ }^{18}$ They then induced stress by hypoxia and phagocytosis by macrophages and observed the functionality of four different TA systems. Antitoxin degradation led to increased toxin transcription. Thus, the results indicated an increase in toxin and antitoxin mRNA. However, the antitoxin, which is unstable, was targeted by a protease, leading to an increase in free toxin. ${ }^{19}$ An increase in toxin expression and a decrease in antitoxin expression were also observed in the present study.

A study by Wang and Wood ${ }^{20}$ evaluated the mqsRA TA system ( $m q s R$ is the antitoxin, and $m q s A$ is the toxin) under oxidative stress and tested its effect on the rpoS gene, which is responsible for controlling 500 genes in Escherichia coli, including biofilm formation. Their findings revealed that the antitoxin ( $m q s R)$ caused a decrease in the expression of the rpoS gene under normal conditions, leaving the E. coli unable to form biofilms. Under oxidative stress conditions, the antitoxin was degraded, and the rpoS gene was induced, resulting in biofilm formation. ${ }^{20}$ Therefore, the results of the current study are consistent with those of Wang and Wood ${ }^{20}$ and indicate that stress can cause antitoxin degradation.

\section{Quantity evaluation of different virulence genes in normal and stress conditions}

The expression levels of the virulence factors of the E. faecalis and E. faecium clinical strains in normal conditions, 


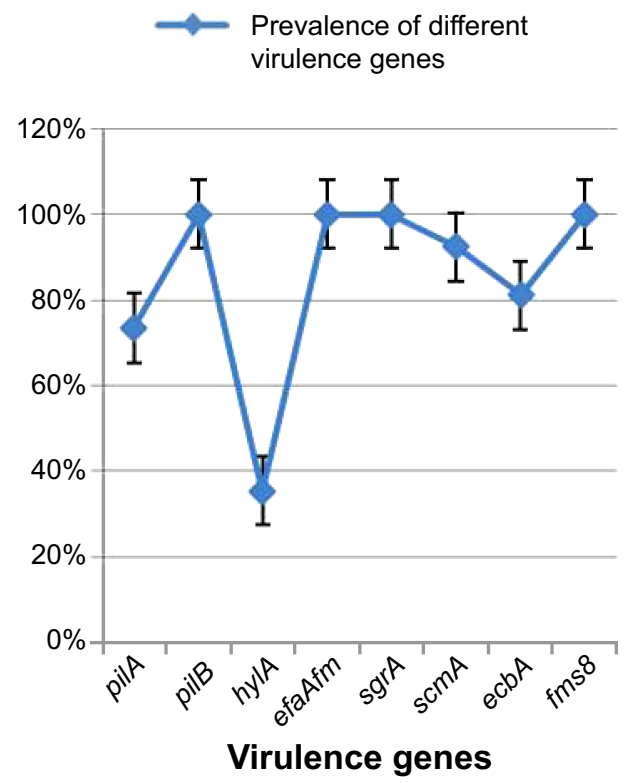

Figure 4 Prevalence of different virulence genes in the clinical Enterococcus faecium and Enterococcus faecalis isolates.

Note: The pilB, fms8, efaAfm, and sgrA genes were identified in all the clinical isolates.

and their response to heat stress, were determined using RTqPCR (Figure 6). As shown in Figure 6, the expressions of all the virulence genes examined in normal conditions were functional, and with heat stress, the expression level of all the virulence genes showed an increase. Therefore, the pathogenicity of E. faecalis and E. faecium might have increased. Only one study has demonstrated the role of stress in the expression level of virulence genes in E. faecalis. There are no studies that explain the expression level of virulence genes under normal and stress conditions in E. faecium. In 2010, Lenz et a ${ }^{21}$ demonstrated that sublethal stress could cause an increase in the pathogenicity of $E$. faecalis by increasing the expression level of virulence genes. E. faecalis were exposed to sublethal osmotic, heat, high hydrostatic pressure, acid, and bile salt stress. Almost the same results were obtained, which indicated that stress causes more pathogenicity in
E. faecalis. The results obtained in the current study were consistent with the findings by Lenz et $\mathrm{al}^{21}$ in that there was an increase in the expression level of all the virulence genes in E. faecalis and E. faecium in the 79 clinical isolates. In the next section, the relationship between virulence genes and TA systems will be discussed.

\section{The mazEFTA system has a direct role in the regulation of virulence genes}

As the mazEF TA locus was prevalent in all clinical strains of E. faecalis and E. faecium, it was targeted for silencing by anti-mazF PNA. Antisense therapy causes the silencing of a specific gene. This technique is applied in molecular bioengineering, therapeutic methods, and antibiotics. ${ }^{9-11}$ PNA is an analog of nucleotide sequences. It has been found to be the best complementary strand of DNA and RNA. ${ }^{12}$ PNA has many properties that make it unique, such as its stability against proteases and nucleases, and its stability in high concentrations of salt and at high temperatures. ${ }^{22}$ Another important property of PNA for efficiency is its size. As normal PNA is larger than an antibiotic, it must be designed to be smaller to pass through the cell. It is also important that the synthesized PNA is efficient against the target gene. The $m a z F$ (antitoxin) is approximately $171 \mathrm{bp}$ in size. The PNA was designed based on the start codon and the ten nucleotides before the start codon region of the $m a z F$ gene. The size of the PNA was 10 mer (shorter than common PNAs). Then, the anti-mazF PNA was evaluated against E. faecium and E. faecalis strains containing the $m a z F$ gene at different concentrations in Mueller Hinton broth. The results were evaluated by RT-qPCR for determination of the specificity of the anti-mazF PNA and its association with the expression level of virulence genes.

As shown in Figure 7, with increases of PNA, the amount of $m a z F$ to be silenced and also the quantity of virulence genes showed a decrease. Therefore, these findings show the direct role of the TA system on the pathogenicity of E. faecium and E. faecalis. PNA antisense therapy is a good technique for

Table I Distribution of the different virulence factors in E. faecium and E. faecalis

\begin{tabular}{llll}
\hline Virulence genes & E. faecalis (50 isolates) & E. faecium (29 isolates) & Total (79 isolates) \\
\hline pilB & $50(100 \%)$ & $29(100 \%)$ & $79(100 \%)$ \\
fms8 & $50(100 \%)$ & $29(100 \%)$ & $79(100 \%)$ \\
efaAfm & $50(100 \%)$ & $29(100 \%)$ & $79(100 \%)$ \\
sgrA & $50(100 \%)$ & $29(100 \%)$ & $79(100 \%)$ \\
scm & $44(88 \%)$ & $29(100 \%)$ & $73(92.4 \%)$ \\
ecbA & $35(70 \%)$ & $29(100 \%)$ & $64(81 \%)$ \\
pilA & $29(58 \%)$ & $29(100 \%)$ & $58(73.4 \%)$ \\
hyl & $4(8 \%)$ & $24(82.7 \%)$ & $28(35.4 \%)$ \\
\hline
\end{tabular}

Abbreviations: E. faecium, Enterococcus faecium; E. faecalis, Enterococcus faecalis. 


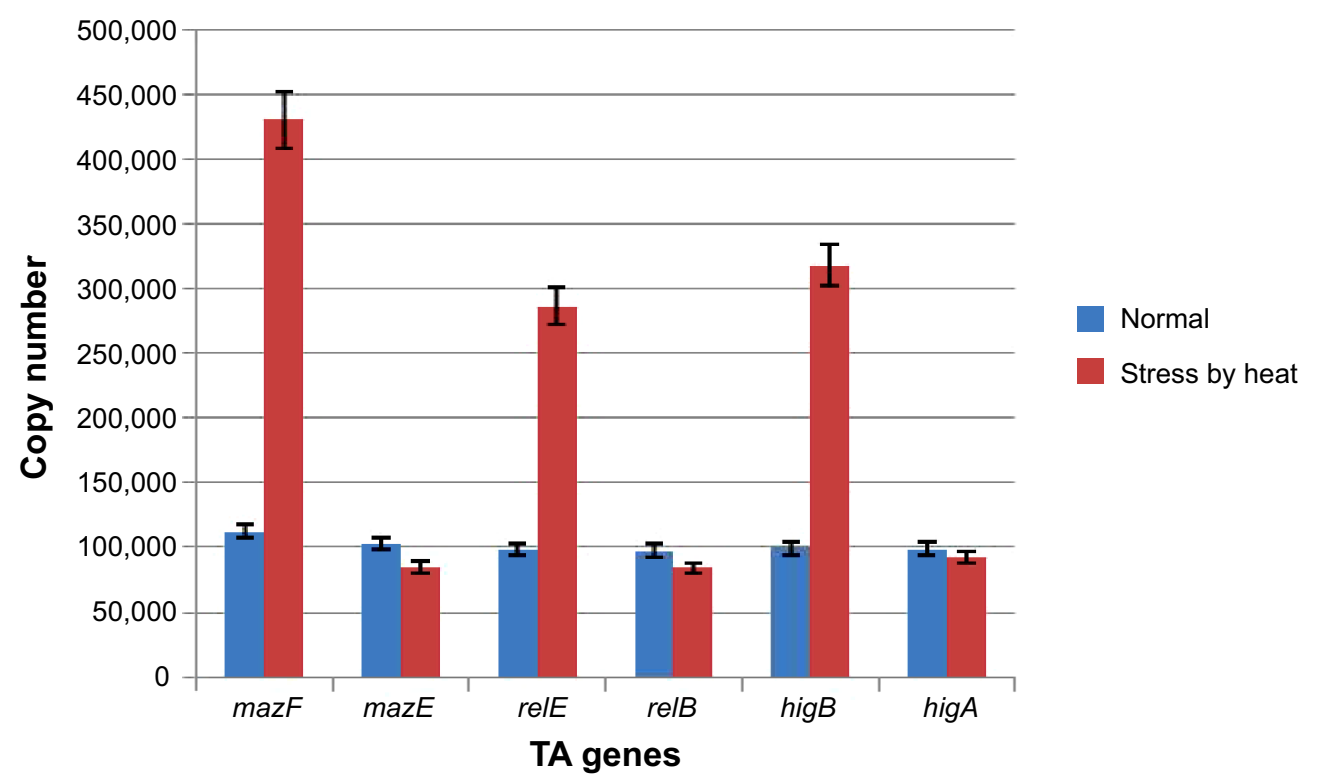

Figure 5 RT-qPCR analysis of the mazEF, relBE, and higBA TA systems under normal and stress conditions.

Notes: Almost the same expression levels for antitoxin and toxin were present in normal conditions, while the levels of toxins were greater than the antitoxins in stress conditions.

Abbreviations: TA, toxin-antitoxin; RT-qPCR, real-time quantitative polymerase chain reaction.

antisense therapy. The results obtained by Good et $\mathrm{al}^{23}$ showed $2 \mu \mathrm{M}$ anti- $a c p P$ PNA SP4 and no viable bacterial cells. Goh et $\mathrm{al}^{24}$ showed the RNA silencing aspect to bacterial growth. They revealed that by reducing the mRNA PNA, a $50 \%$ reduction was observed in the cell growth of E. coli. ${ }^{24}$

Some limitations of this study were the high cost of performing the PNA antisense therapy on a large scale and the higBA TA system.

\section{Conclusion}

This study describes and validates a novel target, $\operatorname{maz} F$, which is found in clinical isolates of E. faecium and E. faecalis strains. First, the mazEF TA locus was shown to be present in all E. faecium strains. We showed that the $m a z E F$ and $h i g B A$ loci are ubiquitous in a collection of $E$. faecium and $E$. faecalis clinical isolates. The expression and function of the mazEF, relBE, and higBA TA loci in

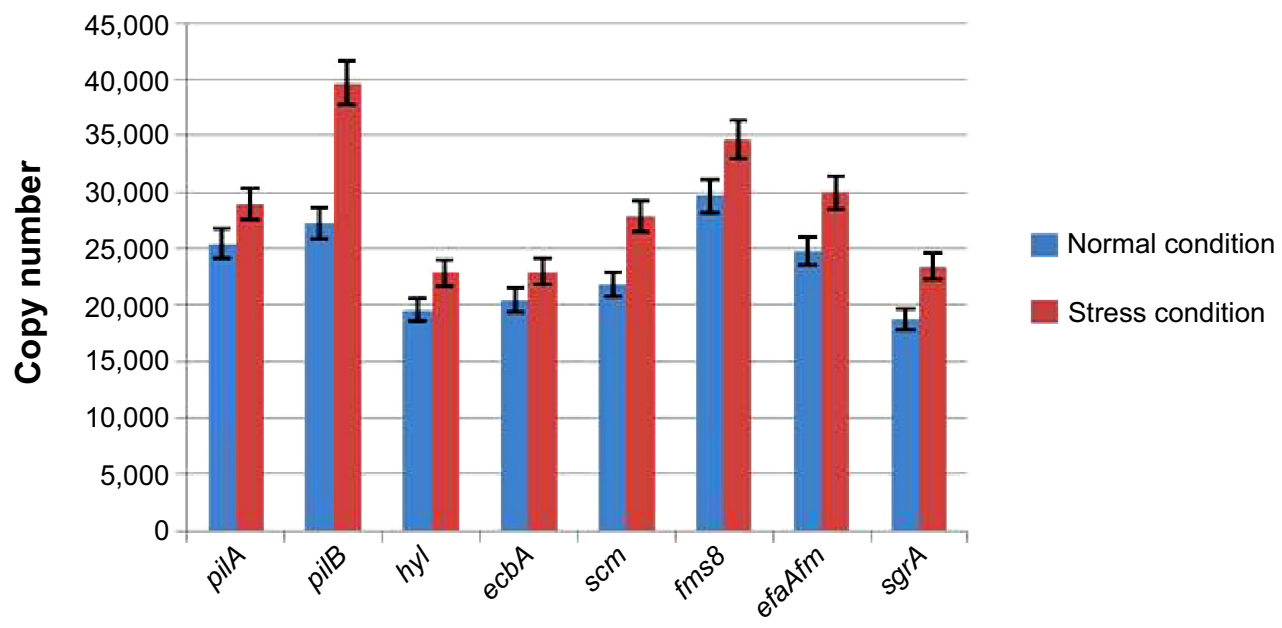

Virulence genes

Figure 6 RT-qPCR analysis of pilA, pilB, hyl, ecbA, scm, fms8, efaAfm, and sgrA virulence genes under normal and stress conditions.

Note: The expression levels of all the virulence genes in heat stress were greater than under normal conditions.

Abbreviation: RT-qPCR, real-time quantitative polymerase chain reaction. 


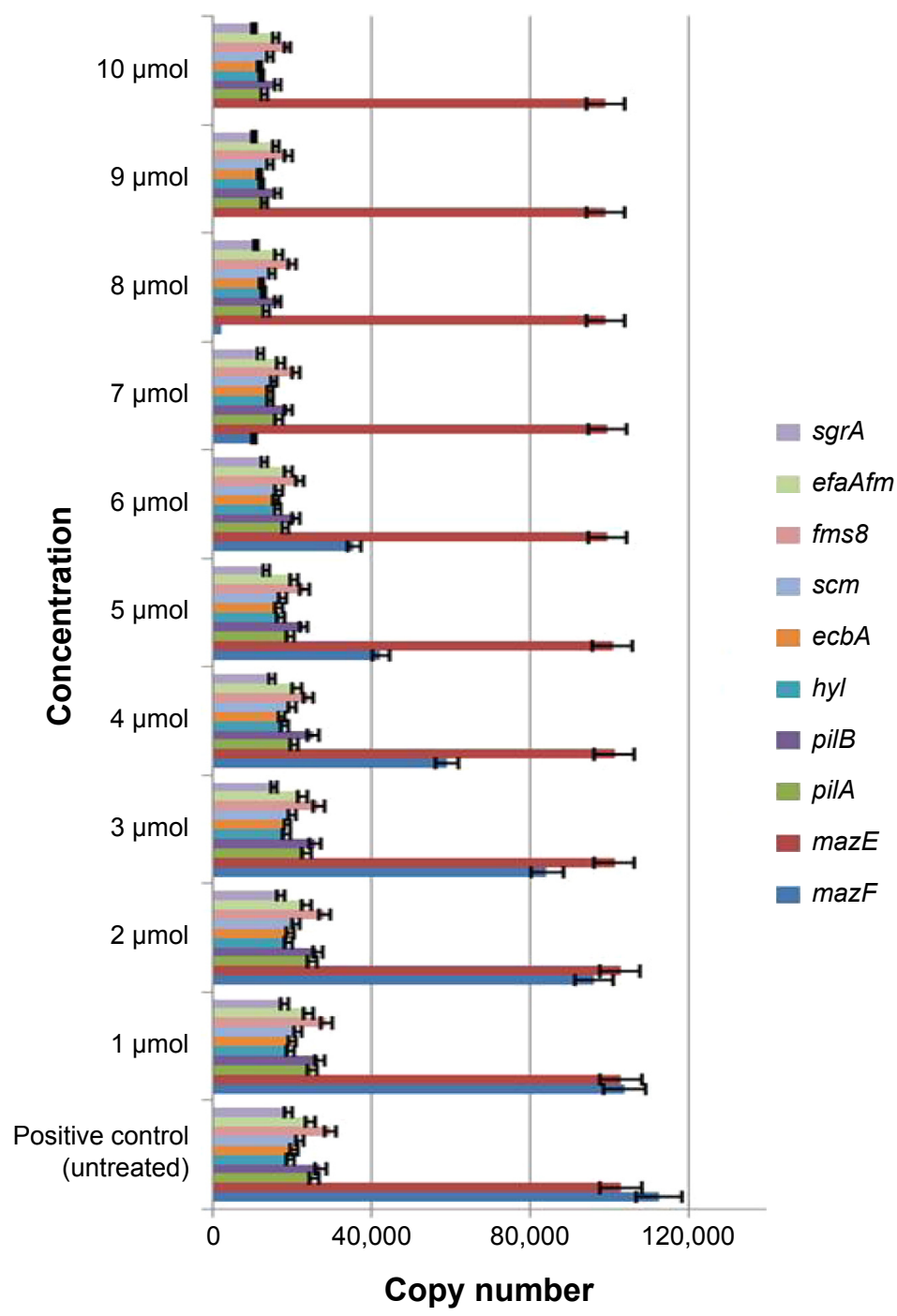

Figure 7 Effect of the anti-mazF PNA against $E$. faecalis and $E$. faecium (the same results were obtained in both) containing the mazEF TA system plasmid. Note: $\mathrm{maz} F$ and all the virulence gene transcripts were subsequently decreased.

Abbreviations: E. faecalis, Enterococcus faecalis; E. faecium, Enterococcus faecium; PNA, peptide nucleic acids; TA, toxin-antitoxin.

E. faecium and E. faecalis strains was confirmed by RTqPCR. Therefore, the TA system appears to be reliably expressed in those strains that pose a particular challenge in medicine. Second, inactivation of the $m a z F$ toxin using an anti-mazF PNA selectively decreased the expression of the virulence genes. Therefore, inactivation of the $m a z F$ toxin has been validated as a novel antimicrobial strategy. This research provides a first step in the introduction of the mazEF TA system as a sensitive target; however, further studies are needed to test the effectiveness of $m a z F$ in vivo. Furthermore, the occurrence and potential for activation of the $m a z E F$ TA system in other pathogenic bacteria remains to be investigated.

\section{Disclosure}

The authors report no conflicts of interest in this work.

\section{References}

1. Van Melderen L, Saavedra De Bast M. Bacterial toxin-antitoxin systems: more than selfih entities? PLoS Genet. 2009;5(3):e1000437.

2. Mine N, Guglielmini J, Wilbaux M, Van Melderen L. The decay of the chromosomally encoded ccdO157 toxin-antitoxin system in the Escherichia coli species. Genetics. 2009;181(4):1557-1566.

3. Mutschler H, Meinhart A. $\varepsilon / \zeta$ systems: their role in resistance, virulence, and their potential for antibiotic development. J Mol Med (Berl). 2011; 89(12):1183-1194.

4. Willems RJ, van Schaik W. Transition of Enterococcus faecium from commensal organism to nosocomial pathogen. Future Microbiol. 2009; 4(9):1125-1135.

5. van Schaik W, Willems RJ. Genome-based insights into the evolution of enterococci. Clin Microbiol Infect. 2010;16(6):527-532.

6. Treitman AN, Yarnold PR, Warren J, Noskin GA. Emerging incidence of Enterococcus faecium among hospital isolates (1993 to 2002). J Clin Microbiol. 2005;43(1):462-463.

7. Sava IG, Heikens E, Huebner J. Pathogenesis and immunity in enterococcal infections. Clin Microbiol Infect. 2010;16(6):533-540.

8. Sillanpää J, Prakash VP, Nallapareddy SR, Murray BE. Distribution of genes encoding MSCRAMMs and Pili in clinical and natural populations of Enterococcus faecium. J Clin Microbiol. 2009;47(4):896-901. 
9. Lee LK, Roth CM. Antisense technology in molecular and cellular bioengineering. Curr Opin Biotechnol. 2003;14(5):505-511.

10. Janson CG, Matthew JD. Peptide Nucleic Acids, Morpholinos and Related Antisense Biomolecules. New York, NY: Springer; 2006.

11. Rasmussen LC, Sperling-Petersen HU, Mortensen KK. Hitting bacteria at the heart of the central dogma: sequence-specifi inhibition. Microb Cell Fact. 2007;6:24.

12. Nielsen PE, Egholm M, Berg RH, Buchardt O. Sequence-selective recognition of DNA by strand displacement with a thymine-substituted polyamide. Science. 1991;254(5037):1497-1500.

13. Jensen KK, Orum H, Nielsen PE, Nordén B. Kinetics for hybridization of peptide nucleic acids (PNA) with DNA and RNA studied with the BIAcore technique. Biochemistry. 1997;36(16):5072-5077.

14. DeNap JC, Hergenrother PJ. Bacterial death comes full circle: targeting plasmid replication in drug-resistant bacteria. Org Biomol Chem. 2005;3(6):959-966.

15. Engelberg-Kulka H, Sat B, Reches M, Amitai S, Hazan R. Bacterial programmed cell death systems as targets for antibiotics. Trends Microbiol. 2004;12(2):66-71.

16. Amitai S, Yassin Y, Engelberg-Kulka H. MazF-mediated cell death in Escherichia coli: a point of no return. J Bacteriol. 2004;186(24): 8295-8300.

17. Anderson KL, Roberts C, Disz T, et al. Characterization of the Staphylococcus aureus heat shock, cold shock stringent, and SOS responses and their effects on log-phase mRNA turnover. J Baceteriol. 2006;188(19):6739-6756.
18. Ramage HR, Connolly LE, Cox JS. Comprehensive functional analysis of Mycobacterium tuberculosis toxin-antitoxin systems: implications for pathogenesis, stress responses, and evolution. PLoS Genet. 2009; 5(12):e1000767.

19. Christensen SK, Mikkelsen M, Pedersen K, Gerdes K. RelE, a global inhibitor of translation, is activated during nutritional stress. Proc Natl Acad Sci U S A. 2001;98(25):14328-14333.

20. Wang X, Wood TK. Toxin-antitoxin systems inflence biofim and persister cell formation and the general stress response. Appl Environ Microbiol. 2011;77(16):5577-5583.

21. Lenz CA, Hew Ferstl CM, Vogel RF. Sub-lethal stress effects on virulence gene expression in Enterococcus faecalis. Food Microbiol. 2010;27(3):317-326.

22. Demidov VV, Potaman VN, Frank-Kamenetskii MD, et al. Stability of peptide nucleic acids in human serum and cellular extracts. Biochem Pharmacol. 1994;48(6):1310-1313.

23. Good L, Awasthi SK, Dryselius R, Larsson O, Nielsen PE. Bactericidal antisense effects of peptide-PNA conjugates. Nat Biotechnol. 2001; 19(4):360-364.

24. Goh S, Boberek JM, Nakashima N, Stach J, Good L. Concurrent growth rate and transcript analyses reveal essential gene stringency in Escherichia coli. PLoS One. 2009;4(6):e6061.
Drug Design, Development and Therapy

\section{Publish your work in this journal}

Drug Design, Development and Therapy is an international, peerreviewed open-access journal that spans the spectrum of drug design and development through to clinical applications. Clinical outcomes, patient safety, and programs for the development and effective, safe, and sustained use of medicines are a feature of the journal, which

\section{Dovepress}

has also been accepted for indexing on PubMed Central. The manuscript management system is completely online and includes a very quick and fair peer-review system, which is all easy to use. Visit http://www.dovepress.com/testimonials.php to read real quotes from published authors.

Submit your manuscript here: http://www.dovepress.com/drug-design-development-and-therapy-journal 